\title{
Taking Action in the Face of Threat: Neural Synchronization Predicts Adaptive Coping
}

\author{
DTatherine A. Collins, ${ }^{1}$ Avi Mendelsohn, ${ }^{1}$ Christopher K. Cain, ${ }^{2,3}$ and Daniela Schiller ${ }^{1}$ \\ ${ }^{1}$ Departments of Psychiatry and Neuroscience, Friedman Brain Institute, Icahn School of Medicine at Mt Sinai, New York, New York 10029, ${ }^{2}$ Emotional \\ Brain Institute, Nathan Kline Institute for Psychiatric Research, Orangeburg, New York 10962, and ${ }^{3}$ Department of Child and Adolescent Psychiatry, New \\ York University School of Medicine, New York, New York 10016
}

The ability to take action in the face of threat is highly diverse across individuals. What are the neural processes that determine individual differences in the ability to cope with danger? We hypothesized that the extent of synchronization between amygdala, striatum, and medial prefrontal cortex (mPFC) would predict successful active coping performance. To test this, we developed a novel computer task based on the principals of Sidman avoidance. Healthy human participants learned through trial and error to move a marker between virtual game board compartments once every $3 \mathrm{~s}$ to avoid mild shocks. Behaviorally, participants exhibited large individual differences. Strikingly, both amygdala-mPFC and caudate-mPFC coupling during active coping trials covaried with final active coping performance across participants. These findings indicate that synchronization between mPFC subregions, and both amygdala and caudate predicts whether individuals will achieve successful active coping performance by the end of training. Thus, successful performance of adaptive actions in the face of threat requires functional synchronization of a neural circuit consisting of mPFC, striatum, and amygdala. Malfunction in the crosstalk between these components might underlie anxiety symptoms and impair individuals' ability to actively cope under stress. This opens an array of possibilities for therapeutic targets for fear and anxiety disorders.

Key words: active avoidance; coping; emotion; fear; individual differences; instrumental learning

\section{Introduction}

Why do some people freeze, whereas others take action in the face of threat? Fear can precipitate stereotyped, involuntary reactions or adaptive safety-seeking actions. People who interrupt a home invasion, for instance, sometimes describe a sense of paralysis, unable to move or speak. Others maintain the capacity for agency and manage to call the police or defend themselves. What are the neural processes that determine individual differences in the ability to cope with danger?

One animal paradigm that captures active coping (AC) is Sidman active avoidance (Sidman, 1953), an effective tool for studying brain regions that differentiate automatic from goaldirected (instrumental) threat responses. In Sidman avoidance, animals are placed in a two-compartment cage where footshocks are scheduled at regular intervals. Initially, animals freeze, expressing an innate defensive reaction. With training, $\sim 80 \%$ of wild-type rodents ("good performers") learn to shuttle between compartments to prevent shock delivery. The others ("poor performers") remain still and continue to expe-

\footnotetext{
Received May 27, 2014; revised Sept. 19, 2014; accepted Sept. 25, 2014.

Author contributions: K.A.C. designed research; K.A.C. performed research; K.A.C. and A.M. analyzed data; K.A.C., A.M., C.K.C., and D.S. wrote the paper.

This work was supported by NIH Grant R21 MH097125 to C.K.C. We thank Mark Baxter, Ifat Levy, and Emily Stern for helpful comments and discussions.

The authors declare no competing financial interests.

Correspondence should be addressed to Dr Daniela Schiller, Department of Psychiatry, One Gustave L Levy Place, Box 1230, New York, NY 10029. E-mail: daniela.schiller@mssm.edu.

DOI:10.1523/JNEUROSCI.2152-14.2014

Copyright $\odot 2014$ the authors $\quad 0270-6474 / 14 / 3414733-06 \$ 15.00 / 0$
}

rience shocks (Myers, 1959). A key feature of this protocol is that action (shuttling) delays shock delivery, allowing control over threats.

Animal studies report that the caudate (Allen, 1972; Allen and Davison, 1973; Delacour et al., 1977; Hart et al., 1978; Wendler et al., 2014), as well as the lateral and basal amygdala (Amorapanth et al., 2000; Choi et al., 2010; Lázaro-Muñoz et al., 2010) contribute to active avoidance. The infralimbic cortex (a probable homolog of human ventromedial prefrontal cortex; vmPFC) facilitates AC by inhibiting central amygdala activity (Moscarello and LeDoux, 2013), thus preventing the expression of stereotyped threat reactions, such as freezing (Choi et al., 2010; LázaroMuñoz et al., 2010; Moscarello and LeDoux, 2013). The brain systems that mediate instrumental threat responses may be conserved (Tricomi et al., 2004; Delgado et al., 2009), but the specific processes that mediate incremental changes in human AC behavior over time, and determine individual differences in performance, are unknown. We hypothesized that the extent of synchronization between amygdala, striatum, and $\mathrm{mPFC}$ would predict successful AC performance.

To test this, we developed a novel computer task based on the principals of Sidman avoidance. Participants learned through trial and error to move a marker between virtual game board compartments once every $3 \mathrm{~s}$ to avoid mild shocks (Fig. 1A). For comparison, AC trials were intermixed with "motor" trials, where participants were instructed to continuously move the game marker and no aversive outcome was delivered. 

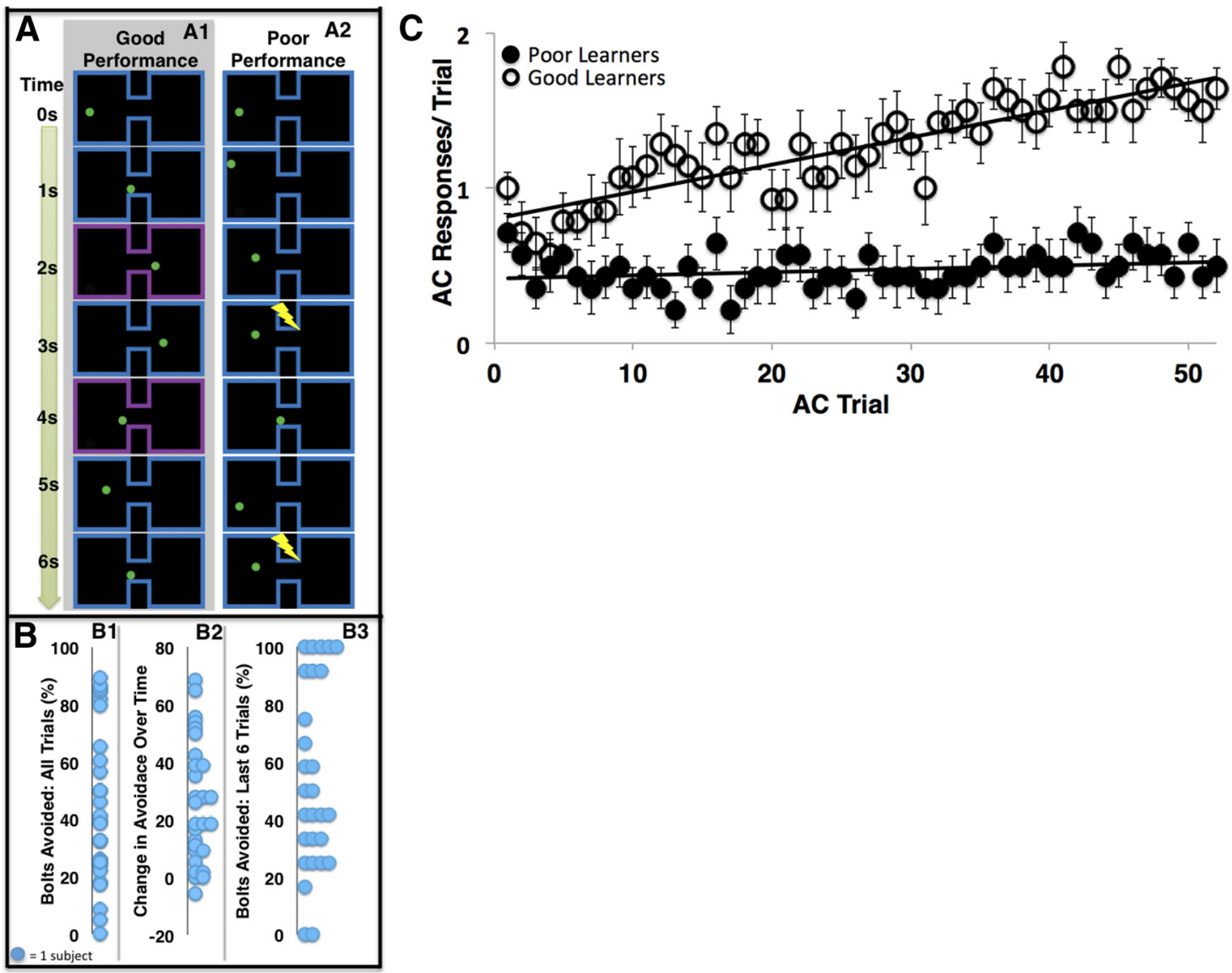

Figure 1. A, Illustration of good and poor performance in a single AC trial. Participants need to move a marker from one side to the other every $3 \mathrm{~s}$ to avoid shock. $\boldsymbol{B}$, Individual differences in $\mathrm{AC}$ performance. Percentage of shocks avoided across 52 trials for each subject $(\boldsymbol{B} 1$, mean $=45.25, S D=5.29)$; change in percentage of shocks avoided in the first versus second half of trials $(\boldsymbol{B 2})$; and percentage of shocks avoided during the last six trials $(B 3$, mean $=6.5, S D=3.86) . C$, $A C$ performance over time in good and poor learners. Mean number of aversive stimuli avoided per trial across $52 \mathrm{AC}$ trials in good learners $(n=14)$ and poor learners $(n=14)$. Subjects classified as good leaners avoided at least $50 \%(6$ of 12$)$ all possible aversive stimuli during the last six $\mathrm{AC}$ trials.

\section{Materials and Methods}

Subjects. Twenty-eight medically and psychiatrically healthy adults (mean age $28.5 \pm 8.1$ years, 14 women) participated in the experiment. The Institutional Review Board of the Icahn School of Medicine at Mount Sinai approved the experimental protocol. All participants provided written informed consent, and were remunerated for their participation.

Self-report forms. Before, during, and after the experimental task participants rated their anxiety using a visual analog scale (Hornblow and Kidson, 1976). After scanning, they completed the State-Trait Anxiety Inventory (STAI; Spielberger, 1983), COPE inventory (Carver et al., 1989), Barrat's Impulsivity Scale (BIS; Patton, 1995), and the Intrinsic Motivation Inventory (IMI) (Ryan, 1983), a scale designed to assess one's internal desire to perform well in a research study. We also administered the third version of the Wide Range Achievement Test's reading subscale (WRAT-III, blue version), a neuropsychological test that estimates the intelligence quotient (IQ); the scores of this scale are significantly correlated with full-scale IQ as assessed by the comprehensive Wechsler Adult Intelligence Scale (Wilkinson, 1993).

Experimental task. We generated a virtual game-board modeled after the cages used in Sidman animal studies (Fig. 1A). The virtual game was constructed using E-prime 2 (Psychology Software Tools) and MATLAB software, composed of two distinct compartments connected by a narrow segment (the "bridge"). Participants used the arrow keys on a traditional keyboard (up, down, left, and right) to move a green marker within the confines of the board during $52 \mathrm{AC}$ and 20 motor control trials. Trials lasted $6 \mathrm{~s}$, separated by jittered intertrial intervals between 4 and $6 \mathrm{~s}$ in length, and began when the game board and marker appeared. The game board was blue or yellow, depending on trial type: AC trials were designated by one of the two colors and motor control trials by the other.
Color assignment (blue AC and yellow motor control trials or vice versa) was pseudorandomized and counterbalanced across subjects. The marker's coordinates at the onset of each trial were randomly selected. The aversive stimulus consisted of a lighting bolt image, which was accompanied by a mild electric shock to the wrist in approximately one-third of trials (18 trials).

We informed participants that they would experience two distinct trial types: AC and motor control trials. Subjects then read the following instructions: "During the (blue/yellow) trials, you may receive electric shocks. You may also see lightning bolt images appear above the board. Your job is to try to avoid the lightning bolts. You can avoid the lightning bolts by moving the green marker around the game board. If you avoid the lightning bolts, you will also avoid the shocks. During the (blue/yellow) trials, you will not receive any shocks or see any lightning bolts. Your job is to move the marker continuously throughout the trial. It is important to keep it moving."

In AC trials, subjects had $3 \mathrm{~s}$ from trial start to move the marker from one board compartment to the other. If they successfully crossed the bridge between compartments, the game board briefly $(200 \mathrm{~ms})$ changed color to purple, signaling effective AC. If they failed to cross the bridge during the first $3 \mathrm{~s}$, a lighting bolt icon appeared on the screen. The participants had to cross the board a second time before the trial ended to avoid a second lightning bolt. If participants remained in one compartment throughout the six-second trial, they executed zero AC responses and experienced two aversive stimuli. If they shuttled across the bridge once, they executed one AC response and experienced one aversive stimulus. If they crossed twice during a single trial, they executed two AC responses and did not experience any aversive stimuli.

In approximately one-third of the AC trials, the lightening bolt was paired with a mild electric shock. We selected this schedule of reinforce- 


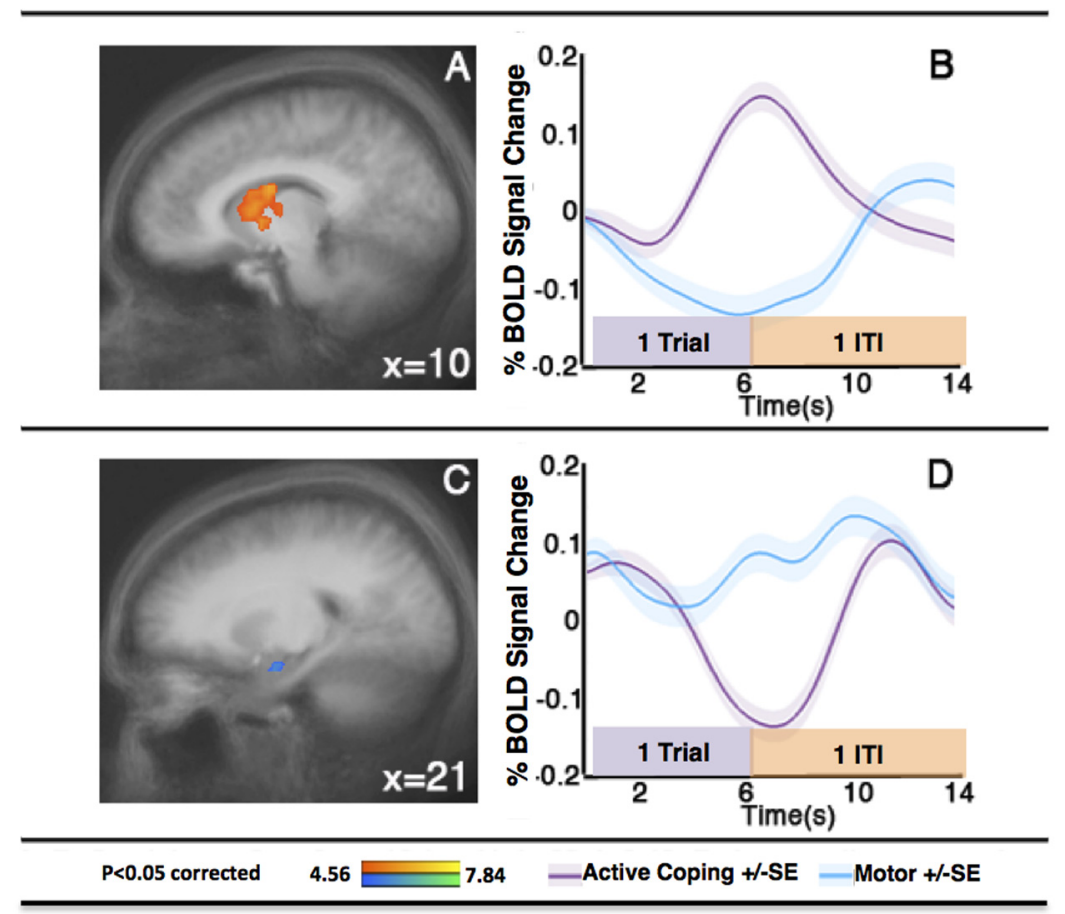

Figure 2. Caudate and amygdala activity during AC versus motor trials. Statistical BOLD activation maps (left column) and event-related averaging of BOLD time courses (right column), in caudate $(\boldsymbol{A}, \boldsymbol{B})$ and amygdala $(\boldsymbol{C}, \boldsymbol{D})$, revealed by the $A C>$ motor contrast. ITI, Intertrial interval.

Table 1. Regions with different patterns of activity during AC compared with motor trials ( $\mathrm{AC}>$ motor)

\begin{tabular}{|c|c|c|c|c|c|c|}
\hline \multirow[b]{2}{*}{ Region } & \multirow[b]{2}{*}{$B A$} & \multicolumn{3}{|c|}{ Talairach } & \multirow[b]{2}{*}{$k$} & \multirow[b]{2}{*}{$t$ (peak } \\
\hline & & $x$ & $y$ & $z$ & & \\
\hline \multicolumn{7}{|l|}{ Active coping $>$ motor } \\
\hline Ranterior insula & 13 & 42 & 12 & 9 & 135 & 7.53 \\
\hline L anterior insula & 13 & -33 & 21 & 9 & 37 & 8.17 \\
\hline RdIPFC & 9 & 46 & 18 & 31 & 105 & 6.67 \\
\hline R caudate & & 10 & 6 & 12 & 83 & 6.43 \\
\hline RPMC & 6 & 4 & 31 & 37 & 39 & 6.13 \\
\hline \multicolumn{7}{|l|}{ Motor $>$ active coping } \\
\hline L mPFC & 10 & -4 & 48 & 2 & 291 & -7.72 \\
\hline L posterior insula & 13 & -47 & -19 & 20 & 139 & -7.50 \\
\hline Rposterior insula & 13 & 34 & -25 & 19 & 19 & -6.32 \\
\hline LPCC & 23 & -3 & -57 & 21 & 247 & -7.44 \\
\hline LIPL & 40 & -36 & -38 & 40 & 92 & -6.61 \\
\hline LdmPFC & 8 & -28 & 15 & 41 & 43 & -6.44 \\
\hline LPHG & 28 & -20 & -24 & -6 & 20 & -5.01 \\
\hline Ramygdala & & 22 & -7 & -7 & 16 & -5.00 \\
\hline LSMA & 6 & -10 & -18 & 51 & 19 & -4.87 \\
\hline
\end{tabular}

R, Right; L, left; dIPFC,dorsolateral prefrontal cortex; PMC, primary motor cortex; $m P F C$, medial prefrontal cortex; $\mathrm{PCC}$, posterior cingulate cortex; IPL, inferior parietal lobule; dmPFC, dorsomedial prefrontal cortex; PHG, parahippocampal gyrus; SMA, supplementary motor area.

ment because it enabled examination of brain activity during AC in the absence of the physiological confound introduced by shock. Partial reinforcement also tends to slow learning rate (Dunsmoor et al., 2007), which allowed us to reveal individual differences. Note that this schedule of reinforcement differs from the one typically used in animal studies of Sidman active avoidance, where animals are shocked every time they fail to shuttle, which might complicate the comparison of our results to those found in rodents.

Before scanning, the experimenter attached a stimulating bar electrode to the participants' nondominant wrist that would deliver the brief (200 $\mathrm{ms})$, mild (10-60 V, 50 pulses/s) stabilized electric shocks from a Grass Medical Instruments stimulator via magnetically shielded and grounded cables during the experiment. Subjects set the intensity of the electric shocks to be administered through a "work-up" procedure. In this pro- cedure, the participant was first given a very mild shock (10 V, $200 \mathrm{~ms}, 50$ pulses/s), which was gradually increased to a level the subject indicated as "uncomfortable, but not painful" (with a maximum level of $60 \mathrm{~V}$ ).

During motor control trials, subjects never experienced any aversive stimuli. By comparing brain activity observed during AC training trials to brain activity observed while subjects processed similar visual stimuli and engaged in similar movements, we aimed to isolate patterns of brain activity related to AC acquisition from that corresponding to perception and physical movement (e.g., in the visual and motor cortices).

fMRI data acquisition. Functional data were acquired on a Siemens Allegra 3.0 tesla scanner during one run, $\sim 14 \mathrm{~min}$ in length, using a single-shot gradient echo T2*-weighted echoplanar imaging sequence (flip angle $=90^{\circ}$, echo time $=35 \mathrm{~ms}$, repetition time $=2000 \mathrm{~ms}$ ) and 36 contiguous transversal interleaved slices with a voxel size of $3 \mathrm{~mm}^{3}$ (field-ofview $=192 \mathrm{~cm}$ ). We also used a T1-weighted magnetization prepared rapid gradient echo protocol (176 sagittal slices, $256 \times 256$ matrix $)$ to record high-resolution $\left(1 \mathrm{~mm}^{3}\right)$ anatomical images.

Behavioral data analysis. Each AC trial was scored as zero, one, or two according to the number of effective AC responses executed. We then calculated, for each participant, the total number of AC responses executed in: all AC trials, the first half of AC trials (1-26), the second half of AC trials (27-52), and the last six AC trials (trials 47-52).

We scored all self-report data and derived subscale scores per the instructions of the instruments' authors. We calculated: "state" and "trait" anxiety summary scores from the STAI; the active coping, planning, suppression, restraint, instrumental social support, emotional social support, positive reinterpretation, acceptance, religion, venting, denial, behavioral disengagement, mental disengagement, and substance use subscales of the COPE; the attention, motor, self-control, cognitive coping, and perseverance subscales, as well as the total score for the BIS; and the interest/enjoyment, perceived competence, perceived choice, and pressure/tension subscales, as well as the total score for the IMI.

We used Pearson correlation and independent samples $t$ tests to explore the relationships between these indices and avoidance behavior. Because we considered all self-report analyses to be exploratory, we used three different measures of avoidance in our correlation analysis: the total number of aversive stimuli executed during the entire task, the total number of aversive stimuli executed in the last six trials, and the difference in the number of aversive stimuli executed in the first half (AC trials $1-27$ ) versus the second half (AC trials 28-54) of avoidance trials.

$f M R I$ data preprocessing. Functional imaging data preprocessing, completed with the BrainVoyager QX software package (Brain Innovation) included: slice scan time correction, voxelwise linear detrending, intensity normalization, high-pass filtering, motion correction, coregistration, normalization to the Talairach template (Talairach and Tournoux, 1998), and three-dimensional smoothing (6 mm fullwidth at half-maximum).

fMRI modeling of brain activity. A first-level general linear model analysis (GLM) was conducted on the blood oxygen level-dependent (BOLD) signal for each subject including three trial-type conditions (AC with lightning bolt and shock, AC with lightning bolt only, and motor) convolved with a standard canonical hemodynamic response function, and six nuisance (motion) regressors, using Neuroelf version 9c (http://neuroelf.net/) and MATLAB software. In second-level analyses, subjects were modeled as a random variable. To examine brain activity related to AC, we used a contrast between AC trials with lightning bolt 

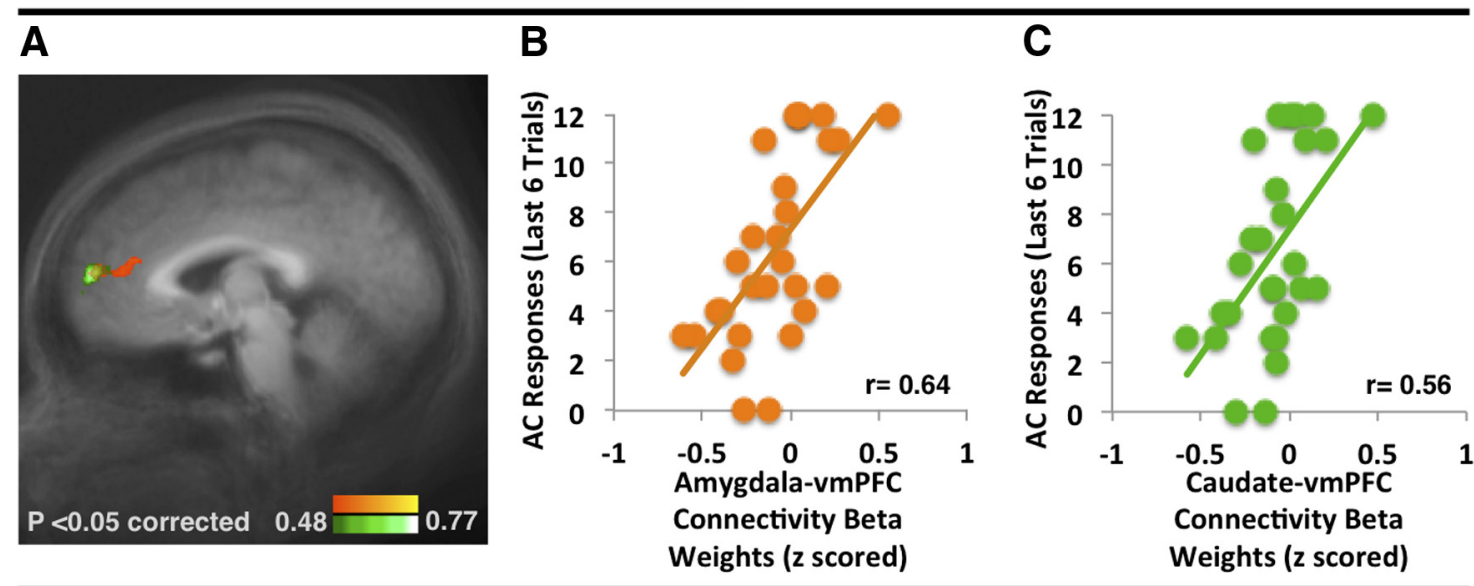

Figure 3. Caudate and amygdala functional connectivity with mPFC during AC trials predict individual differences in final AC performance. Caudate (BA 10, green) and amygdala (BA 32/10, orange) functional connectivity with $\mathrm{mPFC}$ correlated positively with performance in the last six trials $(\boldsymbol{A})$. The scatter plots illustrate the correlation between amygdala-mPFC $(\boldsymbol{B})$ and caudate-mPFC (C) connectivity $\beta$ values and the number of $\mathrm{AC}$ responses in the last six trials.

only and the motor control trials (AC $>$ motor). To correct for multiple comparisons, we used Alphasim as implemented in Neuroelf to identify a cluster size threshold ensuring an FWE rate of $p<0.05$ (Forman et al., 1995; Bennett et al., 2009), corresponding to an uncorrected statistical threshold of $p<0.0001$ and a cluster size threshold of 15 voxels $(405$ $\left.\mathrm{mm}^{3}\right)$.

To determine whether activity in any of the regions-of-interest identified in the $\mathrm{AC}>$ motor contrast predicted $\mathrm{AC}$ performance, we extracted from each region the mean $\mathrm{AC}>$ motor BOLD differential and tested for Pearson correlations with the sum of $\mathrm{AC}$ responses executed in the last six trials.

Analyses of amygdala and caudate functional connectivity. We examined whether the level of functional coactivation between amygdala or caudate with other brain regions during AC trials predicts final AC performance. Specifically, we constructed GLM including the following regressors: AC and motor trials, the time course of caudate or amygdala seed region, and the product of the seed time course and the main AC regressor. This last regressor enabled the estimation of the degree of covariation between the seed's time course and any voxel's time course during AC trials only. We next inserted into the models each subject's final AC score (last 6 trials), and generated second-level statistical maps representing regions that showed significant correlations between final AC scores and seed-functional connectivity values. As before, to correct for multiple comparisons we used an Alphasim-derived cluster threshold to ensure an FWE rate of $p<0.05$. Here, the uncorrected statistical threshold was $p<0.01$ and the cluster size threshold was 77 voxels (2079 $\left.\mathrm{mm}^{3}\right)$.

\section{Results}

\section{Behavior}

Participants exhibited large individual differences in AC behavior (Fig. 1B), successfully avoiding between 0 and $89 \%$ of aversive stimuli. They also exhibited diverse patterns of change in behavior over time: some participants avoided substantially more (up to $68.5 \%$ ) aversive stimuli in the second half of AC trials than in the first half. Others evinced no improvement, or actually performed worse in the later trials. By the end of the experiment, during the last six trials, five participants had mastered the task, executing every possible active coping response in the last six trials. Two participants showed absolutely no evidence of learning, and executed no active coping responses. The remaining participants exhibited various performance levels. Figure $1 C$ depicts AC performance over time in "good" and "poor" learners. Subjects were classified as "good learners" if they avoided at least
$50 \%$ (6 of 12) of all possible aversive stimuli during the last six AC trials.

\section{Self reports}

Subjects reported experiencing more anxiety during the task compared with both before and after the task $\left(F_{(2,20)}=7.31, p<\right.$ $0.005)$. We examined whether the total number of aversive stimuli executed during the entire task, the total number of aversive stimuli executed in the last six trials, or the difference in the number of aversive stimuli executed in the first half (AC trials $1-27$ ) versus the second half (AC trials 28-54) of avoidance trials correlated with any of the self-report scores. None of these correlations survives correction for multiple comparisons. Importantly, IQ was not correlated with AC performance.

\section{Neuroimaging results}

Consistent with previous findings (Delgado et al., 2009; Moscarello and LeDoux, 2013; Wendler et al., 2014), BOLD responses in right caudate were stronger during $\mathrm{AC}$ than motor trials, and right amygdala showed an opposite pattern (Fig. 2; Table 1). Strikingly, both amygdala-mPFC and caudate-mPFC coupling during AC trials (i.e., $\beta$ weights derived from the functional connectivity models) covaried with final AC performance (the number of shocks avoided in the final six trials; Fig. 3). Specifically, the $\beta$ values representing functional connectivity between right caudate and mPFC (BA 10) were positively correlated with final AC performance $\left(r_{(26)}=0.64\right)$. Similarly, $\beta$ values representing functional connectivity between the right amygdala seed and a nearby, more posterior, but partially overlapping, mPFC region (BA 32/ 10) were positively correlated with final AC performance $\left(r_{(26)}=\right.$ 0.52 ). Further, amygdala functional connectivity with the postcentral gyrus (BA 3), a primary somatosensory area, also covaried with final AC performance $\left(r_{(26)}=0.55\right)$.

To validate the specificity of these results, we examined the alternative hypothesis that BOLD activity rather than neural connectivity would predict AC performance. This analysis did not yield any significant results, suggesting that BOLD response amplitude does not readily explain AC behavior.

\section{Discussion}

In the current study, healthy adults completed an AC training during fMRI scanning in which they learned, via trial and error, 
to repeatedly move a virtual marker between two game board compartments to avoid mild electric shocks. We found that the caudate, amygdala, and mPFC were involved in orchestrating AC behavior. As a group, participants displayed more activation in the right caudate and less activation in the right amygdala during AC compared with motor control trials. Synchronization between mPFC subregions and both the amygdala and caudate predicted whether individuals would achieve successful AC performance by the end of training.

We theorize that successful AC performance in the face of threat relies on functional connectivity that synchronizes several essential components: The caudate encodes action-outcome associations (Balleine and Dickinson, 1998; Tricomi et al., 2004; Yin et al., 2005; Lau and Glimcher, 2007; Balleine and O'Doherty, 2010; Sharot et al., 2010), and the anterior mPFC (BA 10) is involved in action selection during instrumental learning (O'Doherty, 2011; Kovach et al., 2012). Thus, interactions between the caudate and anterior MPFC may expedite AC learning by promoting experience-based adaption of behavior. The posterior mPFC (BA 32), in turn, may enable AC by suppressing the central nucleus of the amygdala. A parallel relationship between the infralimbic cortex and the amygdala's central nucleus exists in rodents (Choi et al., 2010; Lázaro-Muñoz et al., 2010; Moscarello and LeDoux, 2013). Central nucleus suppression could prevent the expression of conditioned threat responses (e.g., freezing) that would otherwise interfere with adaptive coping. Central nucleus suppression could also promote signaling in cortical regions that organize and implement instrumental actions (such as the postcentral gyrus; Gozzi et al., 2010).

It should be noted, however, that the mPFC region we observed here (BA32) is dorsal to the one considered the human homolog of the infralimbic cortex (vmPFC, which extends to BA12/25). Human studies supporting this homology have typically used classical fear conditioning and extinction (Roy et al., 2012). It is possible that a more dorsal region relates to aversive instrumental learning in humans. Furthermore, in good performers, we found a positive correlation between activity in this region and amygdala during AC trials, whereas the above explanation would predict an inverse correlation characterized by $\mathrm{mPFC}$ activation and corresponding amygdala suppression. One possibility is that glutamatergic cells in the MPFC project to and stimulate inhibitory (GABAergic) cells in the amygdala (Pinard et al., 2012) that should be more active when amygdala output is suppressed. Alternatively, it is possible that activity in the frontoparietal control network is suppressing amygdala signaling indirectly, with the mPFC acting as mediator (Cole et al., 2013; Spreng et al., 2013). Notably, a recent study identified mPFC GABAergic projections to the striatum and amygdala in mice (Lee et al., 2014). Stimulation of striatal projections elicited acute avoidance behavior, suggesting these inhibitory projections might provide a rapid aversive signal that guides behavior.

The novel AC protocol we developed here is a form of active instrumental learning because threatened subjects had to initiate a specific action to prevent exposure to an aversive stimulus. This differs from passive/inhibitory avoidance where subjects must suppress a prepotent response to prevent exposure to an aversive stimulus, or traditional reward instrumental learning where subjects must initiate a specific action to receive an appetitive stimulus. Our results show that aversive instrumental learning engages many of the same regions engaged by reward instrumental learning, notably the caudate and mPFC. Given the overlap of regions implicated, future studies should directly compare the neural substrates of AC with those of reward-based instrumental learning.

Finally, our results parallel recent work on aversive Pavlovianinstrumental transfer (PIT) in both rats (Campese et al., 2014; McCue et al., 2014) and humans (Nadler et al., 2011; Geurts et al., 2013; Lewis et al., 2013). In aversive PIT, Pavlovian threats facilitate instrumental avoidance, even when Pavlovian conditioning occurs separately. These studies suggest that interactions between PFC striatum, and amygdala mediate aversive PIT, allowing subjects to actively cope with threats that normally trigger passive defensive reactions. Our AC paradigm can be easily adapted to study aversive PIT and potentially reveal subtle differences underlying adaptive versus maladaptive coping responses.

In conclusion, our findings suggest that successful performance of adaptive actions in the face of threat requires ongoing functional synchronization of a neural circuit consisting of mPFC, striatum, and amygdala. Malfunction in the crosstalk between these components might underlie anxiety symptoms and impair individuals' ability to actively cope under stress. This opens an array of possibilities for therapeutic targets for fear and anxiety disorders.

\section{References}

Allen JD (1972) Effects of caudate lesions on the acquisition and retention of sidman avoidance in the rat. Psychonomic Sci 27:157-160. CrossRef

Allen JD, Davison CS (1973) Effects of caudate lesions on signaled and nonsignaled sidman avoidance in the rat. Behav Biol 8:239-250. CrossRef Medline

Amorapanth P, LeDoux JE, Nader K (2000) Different lateral amygdala outputs mediate reactions and actions elicited by a fear-arousing stimulus. Nat Neurosci 3:74-79. CrossRef Medline

Balleine BW, Dickinson A (1998) Goal-directed instrumental action: contingency and incentive learning and their cortical substrates. Neuropharmacology 37:407-419. CrossRef Medline

Balleine BW, O'Doherty JP (2010) Human and rodent homologies in action control: corticostriatal determinants of goal-directed and habitual action. Neuropsychopharmacology 35:48-69. CrossRef Medline

Bennett CM, Wolford GL, Miller MB (2009) The principled control of false positives in neuroimaging. Soc Cogn Affect Neurosci 4:417-422. CrossRef Medline

Campese VD, Kim J, Lázaro-Muñoz G, Pena L, LeDoux JE, Cain CK (2014) Lesions of lateral or central amygdala abolish aversive Pavlovian-toinstrumental transfer in rats. Front Behav Neurosci 8:161. CrossRef Medline

Carver CS, Scheier MF, Weintraub JK (1989) Assessing coping strategies: a theoretically based approach. J Pers Soc Psychol 56:267-283. CrossRef Medline

Choi JS, Cain CK, LeDoux JE (2010) The role of amygdala nuclei in the expression of auditory signaled two-way active avoidance in rats. Learn Mem 17:139-147. CrossRef Medline

Cole MW, Reynolds JR, Power JD, Repovs G, Anticevic A, Braver TS (2013) Multi-task connectivity reveals flexible hubs for adaptive task control. Nat Neurosci 16:1348-1355. CrossRef Medline

Delacour J, Echavarria MT, Senault B, Houcine O (1977) Specificity of avoidance deficits produced by 6-hydroxydopamine lesions of the nigrostriatal system of the rat. J Comp Physiol Psychol 91:875-885. CrossRef Medline

Delgado MR, Jou RL, Ledoux JE, Phelps EA (2009) Avoiding negative outcomes: tracking the mechanisms of avoidance learning in humans during fear conditioning. Front Behav Neurosci 3:33. CrossRef Medline

Dunsmoor JE, Bandettini PA, Knight DC (2007) Impact of continuous versus intermittent CS-UCS pairing on human brain activation during Pavlovian fear conditioning. Behav Neurosci 121:635-642. CrossRef Medline Forman SD, Cohen JD, Fitzgerald M, Eddy WF, Mintun MA, Noll DC (1995) Improved assessment of significant activation in functional magnetic resonance imaging (fMRI): use of a cluster-size threshold. Magn Reson Med 33:636-647. CrossRef Medline

Geurts DE, Huys QJ, den Ouden HE, Cools R (2013) Aversive Pavlovian 
control of instrumental behavior in humans. J Cogn Neurosci 25:14281441. CrossRef Medline

Gozzi A, Jain A, Giovanelli A, Bertollini C, Crestan V, Schwarz AJ, Tsetsenis T, Ragozzino D, Gross CT, Bifone A (2010) A neural switch for active and passive fear. Neuron 67:656-666. CrossRef Medline

Hart T, Chaimas N, Moore RY, Stein DG (1978) Effects of nerve growth factor on behavioral recovery following caudate nucleus lesions in rats. Brain Res Bull 3:245-250. CrossRef Medline

Hornblow AR, Kidson MA (1976) The visual analogue scale for anxiety: a validation study. Aust N Z J Psychiatry 10:339-341. CrossRef Medline

Kovach CK, Daw ND, Rudrauf D, Tranel D, O’Doherty JP, Adolphs R (2012) Anterior prefrontal cortex contributes to action selection through tracking of recent reward trends. J Neurosci 32:8434-8442. CrossRef Medline

Lau B, Glimcher PW (2007) Action and outcome encoding in the primate caudate nucleus. J Neurosci 27:14502-14514. CrossRef Medline

Lázaro-Muñoz G, LeDoux JE, Cain CK (2010) Sidman instrumental avoidance initially depends on lateral and basal amygdala and is constrained by central amygdala-mediated Pavlovian processes. Biol Psychiatry 67: 1120-1127. CrossRef Medline

Lee AT, Vogt D, Rubenstein JL, Sohal VS (2014) A class of GABAergic neurons in the prefrontal cortex sends long-range projections to the nucleus accumbens and elicits acute avoidance behavior. J Neurosci 34:1151911525. CrossRef Medline

Lewis AH, Niznikiewicz MA, Delamater AR, Delgado MR (2013) Avoidance-based human Pavlovian-to-instrumental transfer. Eur J Neurosci 38:3740-3748. CrossRef Medline

McCue MG, LeDoux JE, Cain CK (2014) Medial amygdala lesions selectively block aversive Pavlovian-instrumental transfer in rats. Front Behav Neurosci 8:329. CrossRef

Moscarello JM, LeDoux JE (2013) Active avoidance learning requires prefrontal suppression of amygdala-mediated defensive reactions. J Neurosci 33:3815-3823. CrossRef Medline

Myers AK (1959) Avoidance learning as a function of several training conditions and strain differences in rats. J Comp Physiol Psychol 52:381-386. CrossRef Medline

Nadler N, Delgado MR, Delamater AR (2011) Pavlovian to instrumental transfer of control in a human learning task. Emotion 11:1112-1123. CrossRef Medline

O’Doherty JP (2011) Contributions of the ventromedial prefrontal cortex to goal-directed action selection. Ann N Y Acad Sci 1239:118-129. CrossRef Medline

Patton JH, Stanford MS, Barratt ES (1995) Factor structure of the Barratt impulsiveness scale. J Clin Psychol 51:768-774. CrossRef Medline

Pinard CR, Mascagni F, McDonald AJ (2012) Medial prefrontal cortical innervation of the intercalated nuclear region of the amygdala. Neuroscience 205:112-124. CrossRef Medline

Roy M, Shohamy D, Wager TD (2012) Ventromedial prefrontal-subcortical systems and the generation of affective meaning. Trends Cogn Sci 16:147156. CrossRef Medline

Ryan RM (1983) Relation of reward contingency and interpersonal context to intrinsic motivation: A review and test using cognitive evaluation theory. J Pers Soc Psychol 45:736-750. CrossRef

Sharot T, Shiner T, Dolan RJ (2010) Experience and choice shape expected aversive outcomes. J Neurosci 30:9209-9215. CrossRef Medline

Sidman M (1953) Avoidance conditioning with brief shock and no exteroceptive warning signal. Science 118:157-158. CrossRef Medline

Spielberger CS (1983) Manual for the state-trait anxiety inventory. Palo Alto, CA: Consulting Psychologist.

Spreng RN, Sepulcre J, Turner GR, Stevens WD, Schacter DL (2013) Intrinsic architecture underlying the relations among the default, dorsal attention, and frontoparietal control networks of the human brain. J Cogn Neurosci 25:74-86. CrossRef Medline

Talairach J, Tournoux P (1998) Co-planar stereotaxic atlas of the human brain: an approach to medical cerebral imaging. New York: Thieme.

Tricomi EM, Delgado MR, Fiez JA (2004) Modulation of caudate activity by action contingency. Neuron 41:281-292. CrossRef Medline

Wendler E, Gaspar JC, Ferreira TL, Barbiero JK, Andreatini R, Vital MA, Blaha CD, Winn P, Da Cunha C (2014) The roles of the nucleus accumbens core, dorsomedial striatum, and dorsolateral striatum in learning: performance and extinction of Pavlovian fear-conditioned responses and instrumental avoidance responses. Neurobiol Learn Mem 109:27-36. CrossRef Medline

Wilkinson GS (1993) Wide range achievement test: WRAT 3. Wilmington, DE: Wide Range.

Yin HH, Ostlund SB, Knowlton BJ, Balleine BW (2005) The role of the dorsomedial striatum in instrumental conditioning. Eur J Neurosci 22: 513-523. CrossRef Medline 